\title{
Acetaminophen Induced Transient Hypothermia in Pediatrics Population Undergoing General Anesthesia; a Double Blinded Randomized Controlled Clinical Trial
}

\author{
Makhoul JG and Kafrouni HF* \\ Department of Anesthesiology, University of Balamand, Saint George Hospital University \\ Medical Center, Lebanon
}

*Corresponding author: Hazem F.Kafrouni, Assistant Professor of Clinical Anesthesia,
Department of Anesthesiology, Saint George Hospital University Medical center, University
of Balamand, St. George Health Complex, Youssef Surock Street, Achrafieh 1100 2807, Beirut,
LEBANON; Tel: 0096170131370, 009611441000; Email: hfkafrouni@stgeorgehospital.org

\section{Research Article}

Volume 6 Issue 2

Received Date: August 05, 2021

Published Date: September 02, 2021

DOI: $10.23880 /$ accmj-16000194

\section{Abstract}

Background: Perioperative hypothermia has been strongly associated with poor outcomes in children undergoing general anesthesia.

Methods: This study consisted of a monocentric, block randomized, double blinded, placebo-controlled clinical trial over a period of 3 months at a tertiary referral center. Non-febrile patients with no significant medical history younger than 3 years and having surgery for a minimum duration of one hour under General Anesthesia were chosen. Thirty minutes prior to emergence, patients received either IV Acetaminophen (Group P) or a placebo solution of normal saline (Group N). The temperature was recorded at $\mathrm{T} 0, \mathrm{~T} 15, \mathrm{~T} 30, \mathrm{~T} 45$ and $\mathrm{T} 60$ intervals.

Results: 60 out of 84 patients were enrolled, the sample consisted of 58.3\% (n=35) males and $41.7 \%(n=25)$ females with a mean age $1.16 \pm 0.92$ years. When using a t test to compare the mean core temperature, at a $95 \%$ Confidence Interval and $\pm=0.05$, data showed that in Group P $\left(35.52 \pm 0.68 \mathrm{oC}<36^{\circ} \mathrm{C}\right)$ and in Group N $\left(36.07 \pm 0.71 \mathrm{oC}>36^{\circ} \mathrm{C}\right)$ were significantly different at $\mathrm{T} 30(\mathrm{p}<0.005)$. A similar significance was observed at $\mathrm{T} 45$ yet not at $\mathrm{T} 60$.

Conclusion: Acetaminophen seems to have induced a significant transient hypothermia at T30 in the pediatric population, which coincides in this study with the critical time of emergence. At T60, no significant difference was observed, suggesting that patients' core body temperature values returned to normality. Therefore, it is recommended to administer Acetaminophen at least 60 minutes prior to emergence to avoid a core temperature drop during this critical phase.

Keywords: General Anesthesia; Pediatrics; Acetaminophen; Hypothermia

Glossary of Terms: IV: Intravenous; GA: General Anesthesia; $T_{c}$ : core body temperature; PACU: PostAnesthesia Care Unit; IPH: Inadvertent Perioperative Hypothermia; VIMA: Volatile Induction of Anesthesia; ${ }^{\circ} \mathrm{C}$ : Degrees Celsius; T0: time of administration of solution, 30 minutes before expected emergence; T15: 15 minutes after solution administration; T30: 30 minutes after solution administration, coinciding with emergence; T45: 45 minutes after solution administration; T60: 60 minutes after solution administration.

\section{Introduction}

Acetaminophen, also known as Paracetamol, is a commonly prescribed analgesic for the treatment of acute pain. Regularly used and appreciate in the pediatrics population, its efficacy and safety, particularly in IV formulations are still unclear [1]. The mechanism by which Acetaminophen works is not precisely known yet, thought to be mediated centrally. Its analgesic effect starts at approximately fifteen minutes after administration, peaks at 


\section{Anaesthesia \& Critical Care Medicine Journal}

approximately one hour, and lasts for four to six hours, while its antipyretic effect begins within thirty minutes [2].

Although Acetaminophen is generally considered a safe drug, some adverse effects have been reported, especially when administered in higher than recommended doses. The most common adverse effects (incidence of at least 5\%) in the pediatrics population are pruritus, agitation, atelectasis, constipation, vomiting, and nausea [2]. Some cases reported hepatotoxicity that occurs with recommended dosages in children; other cases reported liver failure [3]. In the malnourished, hepatotoxicity may occur at lower doses of IV Acetaminophen compared to oral forms [4]. Another undesirable side effect of Acetaminophen is hypothermia; in a specific case study, it has been shown that four pediatrics cases presented with hypothermia after an administration of normal therapeutic doses of Acetaminophen [5]. As a definition, Inadvertent Perioperative Hypothermia (IPH) is described as core body temperature $\left(T_{\mathrm{c}}\right)$ below $36^{\circ} \mathrm{C}[6]$. As thermoregulation is very crucial and has significant consequences, especially in the pediatrics population, preventing hypothermia should be a serious concern. Some of the major consequences of hypothermia include patient discomfort, postoperative cardiovascular events, coagulopathies, vasoconstriction, higher risk of surgical wound infection, delay in drug metabolism, sympathetic stimulation, misinterpretation of arterial blood gas, and increased length of hospital stay and Post Anesthesia Care Unit (PACU) recovery time [6-9]. A mild degree of perioperative hypothermia can be associated with significant morbidity and mortality [10].

Emergence from General Anesthesia (GA) is a process with a gradual return of consciousness after discontinuing anesthetic and adjuvant agents. Transition from a surgical anesthesia state to an awake one with intact protective reflexes is usually smooth for most patients [6]. When delayed emergence occurs, differential diagnoses include surgical complications, neurologic squeal, endocrine disturbances, and metabolic derangements [11]. Among the metabolic causes, hypothermia constitutes an important factor in delaying emergence, hence preventing it is critical. In the light of these findings, this study aims to assess the effect of intra-operative weight adapted doses of IV Acetaminophen in inducing hypothermia in the pediatric population undergoing GA.

\section{Methods}

The study consisted of a monocentric, randomized, placebo-controlled clinical trial. Approval from the Institutional Review Board was obtained. This paper was registered by Dr. H.K the principal investigator, at ClinicalTrials.gov number NCT04608669, verified by the registry in June 2019, prior to patient enrolment. This manuscript adheres to the applicable CONSORT guidelines. Throughout the study, patients' confidentiality is respected.

Pediatrics patients from both genders and less than 3 years of age, presenting to our tertiary referral center between July 2019 and September 2019 to undergo surgery under GA, with a minimum operative time of sixty minutes, were chosen. Patients were to have no significant past medical history and no fever at the time of admission or surgery, with the necessity to use an intra-rectal temperature probe. Member of the research team selected the patients during the pre-operative anesthesia examination. Patients were enrolled only after obtaining, preoperatively, a written consent from their parents or legal caregivers; all the necessary information about the study, risks and benefits were explained.

In the setting of each surgery, the operating room temperature was fixed at $23^{\circ} \mathrm{C}$. Underbody warming achieved by forced air warmers where similar intra-operative body warming, and fluid warming guidelines were provided to all patients and remained unchanged until reaching the PACU. $T_{c}$ was measured on one-minute interval using an intrarectal temperature probe, wired to a case-by-case calibrated temperature sensor of a Philips IntellVue MX700 bedside patient monitor, both intra-operatively and postoperatively in the PACU. In addition, anesthesia drugs and procedures provided were the same to all patients; all were subject to volatile induction of anesthesia (VIMA) followed by the administration of appropriate doses of Rocuronium and Fentanyl, while anesthesia was maintained by continuous Sevoflurane inhalation. Regional Anesthesia was not performed for these patients in the setting of GA.

By blocks of three, patients were allocated to either Group P (receiving Acetaminophen) or Group N (receiving the Placebo of Normal Saline solution). Well-informed of the case's group allocation, Physician A reassessed the temperature in the induction room, reviewed the case, registered the age and weight, and accordingly prepared a solution of Acetaminophen or Placebo. The Acetaminophen solution was prepared according to the department guideline; weight adjusted dose: $7.5 \mathrm{mg} / \mathrm{kg}$ for patients less than 6 months of age; $15 \mathrm{mg} / \mathrm{kg}$ for those aged between 6 months and 3 years. The solution was then provided without indication of its content to Physician B, keeping that physician and the patient blinded. As per the departmental protocol, the solution was administrated thirty minutes before the expected time for emergence from GA (T0). Physician B registered the temperature values at a fifteenminute interval, first in the operating room, starting at T0 (time of administration), T15 (15 minutes), T30 (30 minutes; emergence from anesthesia), then in the PACU at T45 (45 
minutes) and T60 (60 minutes).

\section{Statistical Analysis}

Subjects were enrolled sequentially over a period of three months and allocated by the data collection team by blocks of three into either Groups P or N. This block allocation achieved randomization and decreased the disparity between the group sizes. All results were handed to the data collection team and entered anonymously into Excel Sheets. The cutoff for hypothermic values is, as defined by IPH, a $T_{c}$ below $36.0^{\circ} \mathrm{C}$. After data collection, data analysis was performed using the SPSS software; mean and standard deviations were calculated, normalization test and $t$ tests were performed to compare $T_{c}$ means at different times between the $\mathrm{P}$ and $\mathrm{N}$ groups, at $95 \%$ Confidence Interval and $\alpha=0.05$.

\section{Results}

Over a period of 3 months, 84 patients aged less than 3 years presenting for a surgery under GA, with a minimum operative time of sixty minutes were assessed during the pre-operative examination. Out of the 84 patients, 24 were excluded due to the lack of consent $(n=6)$ or for having either fever or previous medical conditions $(n=18)$, thus 60 patients were enrolled (Figure 1). Because the subjects entered sequentially, and randomization was done in blocks, Groups P and N both ended with a sample size of 30. No intraoperative complications occurred requiring elimination of any subjects.

In this study, the mean age was $1.16 \pm 0.92$ years, while males represented $58.3 \%(n=35)$ and females $41.7 \%(n=25)$ (Table 1). The temperature variation for each group was noted, and the means by group for $\mathrm{T} 0, \mathrm{~T} 15, \mathrm{~T} 30, \mathrm{~T} 45$, and T60 was calculated (Table 2 and Figure 2). In Group P, at T30 $\left(35.52 \pm 0.68{ }^{\circ} \mathrm{C}\right)$ and $\mathrm{T} 45\left(35.71 \pm 0.82{ }^{\circ} \mathrm{C}\right)$ most values are below the cutoff of $36.0^{\circ} \mathrm{C}$ reflecting a state of IPH.

Using SPSS, data was found to have normal distribution without outliers. In order to compare $T_{c}$ means at different times between the $\mathrm{P}$ and $\mathrm{N}$ groups, $t$ tests were performed (Table 2). At T0, no significant difference between the means of Group $\mathrm{P}\left(36.31 \pm 0.58{ }^{\circ} \mathrm{C}\right)$ and $\mathrm{N}\left(36.17 \pm 0.78{ }^{\circ} \mathrm{C}\right)$ was observes $(p>0.05)$, as it was the case at T15 $(p>0.05)$. Whereas at T30, the mean temperature of Group P $\left(35.52 \pm 0.68^{\circ} \mathrm{C}\right)$ and that of Group $\mathrm{N}\left(36.07 \pm 0.71^{\circ} \mathrm{C}\right)$ were significantly different $(p<0.05)$. Similar significant findings were observed at T45, however, at T60, no significant difference was found between the means $(p>0.05)$.

Post-operative pain was the outmost harm caused by this study in Group $\mathrm{N}$ where Acetaminophen was not administered. However, intraoperative Fentanyl was given, and Physician A administered weight adjusted Acetaminophen or Nalbuphine in this group after the study time elapsed.

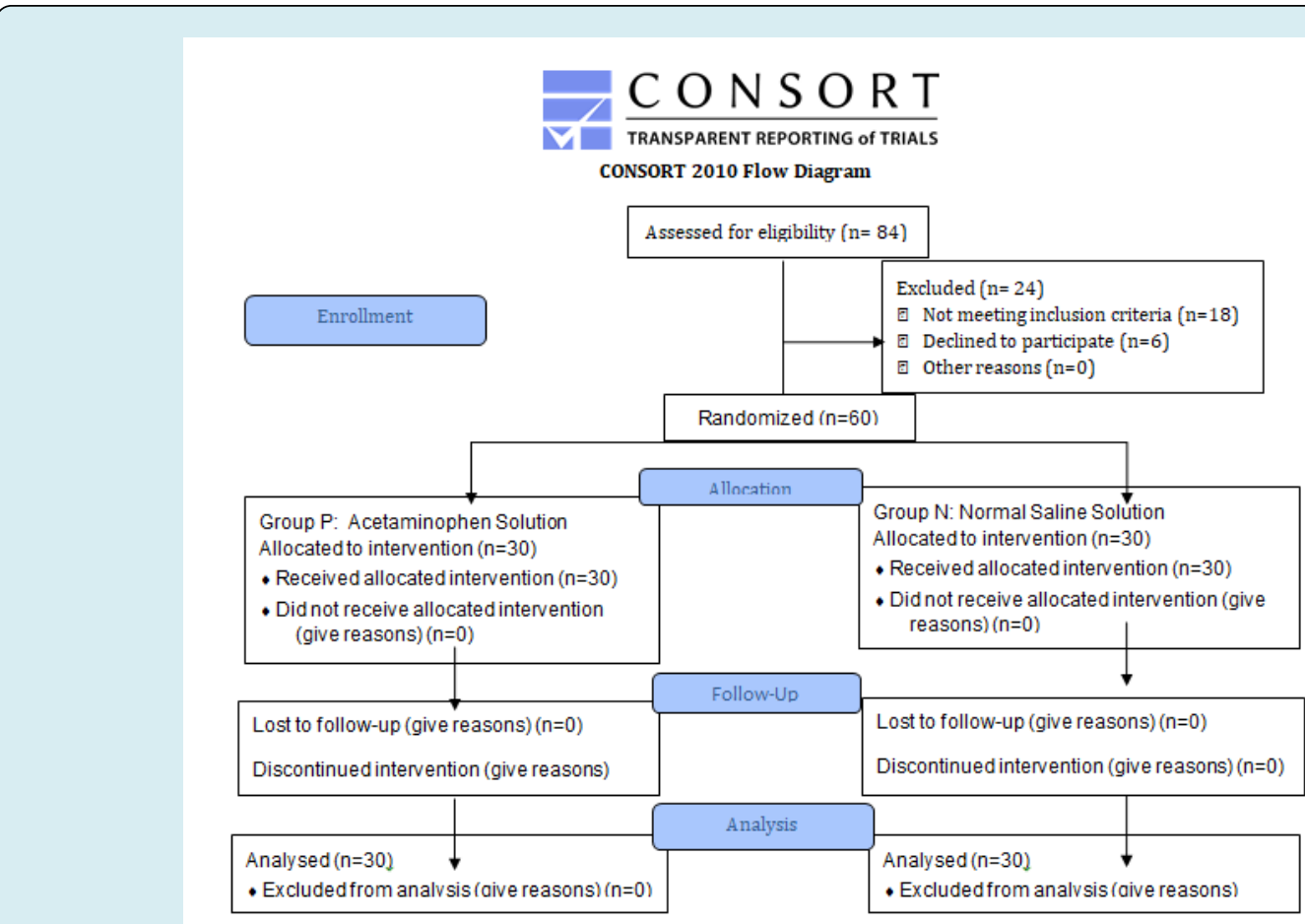

Figure 1: Consort Flow diagram illustrating the steps of enrollment, allocation, follow-up and analysis. 


\section{Anaesthesia \& Critical Care Medicine Journal}

Variation of Temperature $\left(\mathrm{in}^{\circ} \mathrm{C}\right)$ in Group $\mathrm{P}$ and $\mathrm{N}$ after solution administration at T0

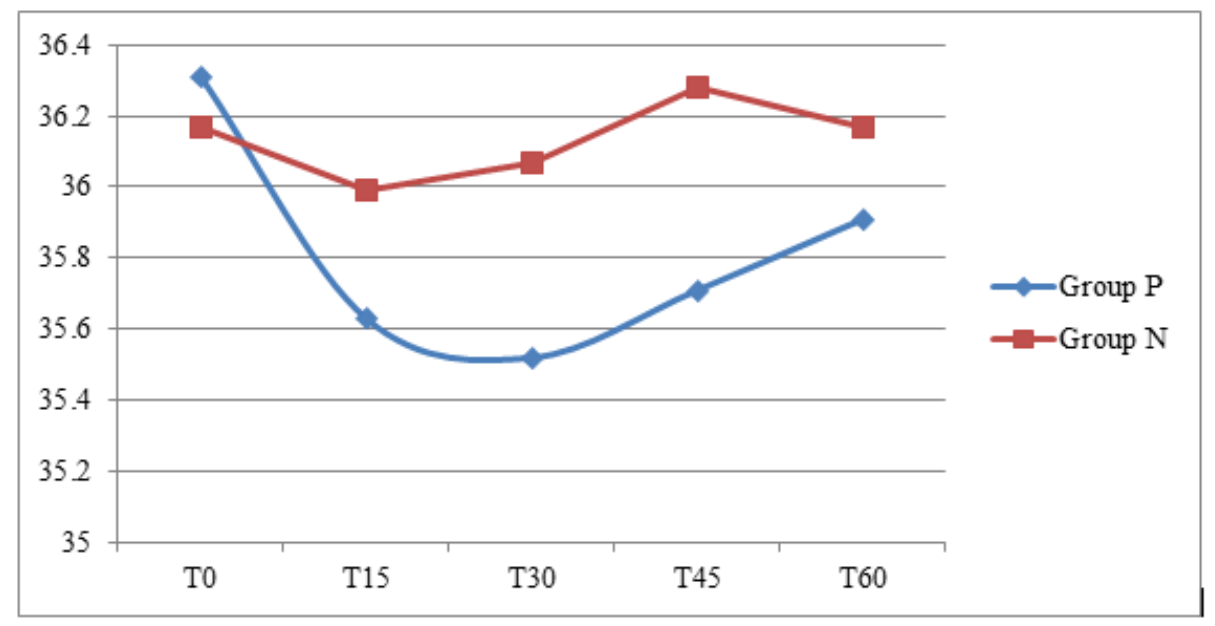

Figure 2: Variation of mean $T_{c}$ (core body temperature in ${ }^{\circ} \mathrm{C}$ ) in Group $\mathrm{P}$ and $\mathrm{N}$ during one hour after solution administration at T0, with fifteen minutes interval measurements at T15, T30, T45 and T60 minutes.

\begin{tabular}{|c|c|c|c|}
\hline \multicolumn{4}{|c|}{ Table 1: Data Distribution } \\
\hline Parameter & Group P & Group N & Total \\
\hline Number of subjects & $n=30$ & $n=30$ & $n=60$ \\
\hline Male Sex & $56.7 \%(n=17)$ & $60 \%(n=18)$ & $58.3 \%(n=35)$ \\
\hline Female Sex & $43.3 \%(n=13)$ & $40 \%(n=12)$ & $1.16 \pm 0.92$ \\
\hline
\end{tabular}

Table 1: Data Distribution showing the percentages of males and females included in the study groups in addition to comparison of gender and mean age (in years) between groups $\mathrm{P}$ and $\mathrm{N}$.

\begin{tabular}{|c|c|c|c|}
\hline & & & \\
\hline & & & \\
\hline T15 & $35.63 \pm 0.63$ & $35.99 \pm 0.75$ & 0.051 \\
\hline T30 & $35.52 \pm 0.68$ & $36.07 \pm 0.71$ & 0.003 \\
\hline T45 & $35.71 \pm 0.82$ & $36.28 \pm 0.72$ & 0.006 \\
\hline T60 & $35.91 \pm 0.89$ & $36.17 \pm 1.97$ & 0.501 \\
\hline \multicolumn{4}{|c|}{ Abbreviations: $T_{c}$ core body temperature ; ${ }^{\circ} \mathrm{C}$, degrees Celsius } \\
\hline
\end{tabular}

Table 2: Mean Temperature in ${ }^{\circ} \mathrm{C}$ and $t$ test $p$ value at $95 \%$ Confidence Interval.

\section{Discussion}

The hypothermic effect of Acetaminophen was proven to be due to its effect on the GABA receptors in the brain $[12,13]$. However, as occurrences of hypothermia are usually of low or moderate severity, it is likely that it is under reported by clinicians [14]. With an unknown mechanism of action, Acetaminophen may worsen perioperative hypothermia when combined with the known hypothermic effects of GA.
Despite not causing hypothermia in awake normothermic patients, it may predispose patients to a greater degree of perioperative hypothermia when combined with GA $[15,16]$. ASA statement has reported the impact of multiple warming modalities on perioperative $T_{c}$ in the pediatric patients under GA, providing evidence that the active warming modalities used in this study (forced-air warming blankets) concur with standards-of-care [17]. Many negative implications of 


\section{Anaesthesia \& Critical Care Medicine Journal}

IPH causing increased risk of surgical site infection, delayed healing, increased bleeding and transfusion requirements and increased rate of cardiac events have all been implicated and have serious consequences especially in the pediatrics population [18-21].

Acetaminophen induced hypothermia in the pediatrics population at emergence from GA was investigated by comparing 2 groups of children aged less than 3 years, where randomly 30 patients received weight adapted doses of Acetaminophen Solution (Group P) while 30 others received a Placebo Solution (Group N).

This article found that IV acetaminophen induces a statistically significant decrease in $T_{c}$. Despite the same thermoregulation precautions taken in both groups, the mean $T_{\mathrm{c}}$ in Group P at T30 (35.52 \pm 0.68$)$ and T45 (35.71 $\pm 0.82)$ are both bellow the cutoff value of IPH $\left(36.0^{\circ} \mathrm{C}\right)$ unlike in Group N (Figure 2). At T30, corresponding to the time of emergence from GA, and T45, the mean $T_{c}$ of Group P and Group N are significantly different ( $p<0.05$ at T30 and T45). This means that a significant temperature decrease reaching hypothermia is induced starting 30 minutes after Acetaminophen administration that continues to be significant as compared to the control Group $\mathrm{N}$ at 45 minutes post administration. However, at T60, no significant difference is found between the means ( $p>0.05)$. Therefore, at $\mathrm{T} 60$, the mean temperature of Group P returns to normality as shown by the convergence of the two curves in Figure 2.

The significance of these findings suggests that Acetaminophen administration at least 60 minutes prior to emergence is important for avoiding a $T_{c}$ drop during this critical phase. Considering the undesired side effects of hypothermia at emergence from GA, and based on the result of this study, two options seem feasible to avoid these undesirable adverse effects. First option would be administering Acetaminophen after emergence which would necessitate PACU monitoring for hypothermia, where however, effective monitoring cannot always be guaranteed. The second option would be to administer acetaminophen one hour prior to emergence so that normothermia would be re-attained at the time of emergence (Figure 2). This will avoid having hypothermia at emergence while still benefiting from the optimal analgesic effects of Acetaminophen.

Those results were consistent with previous studies where Acetaminophen administration induced a transient decrease in $T_{c}$. Most investigations were carried out on adult population and very few in the pediatrics. In a review of Acetaminophen's clinical pharmacology in neonates, while discussing thoroughly many of this molecule's effect, hypothermia was barely mentioned [22]. This shows the insufficient research done in the investigation of Acetaminophen's hypothermic effect in the pediatrics population [23].

In a trial investigating the hypothermic effects of Acetaminophen in the absence of fever in healthy adults, it was found that acetaminophen-induced hypothermia is significantly exacerbated during cold stress but has no effect on $T_{c}$ in a thermo-neutral environment, therefore; Acetaminophen induced hypothermia is strongly influenced by the ambient temperature during which it is administered [24]. This mild hypothermia may be explained by the IPH that is a common consequence of GA [18]. Another study demonstrated that acute Acetaminophen ingestion at a dose of $20 \mathrm{mg} / \mathrm{kg}$ lean body mass reduces $T_{\mathrm{c}}$ in non-febrile normothermic humans during a $120 \mathrm{~min}$ passive exposure to $20^{\circ} \mathrm{C}$ [24]. However, in one study, acetaminophen administration in neonates with normothermia had no significant effect on $T_{\mathrm{c}}$ and no hypothermia was reported on the contrary to both children and adults [22].

A research study investigating whether IV acetaminophen may worsen IPH when combined with GA for adult total hip arthroplasties found that Acetaminophen does not appear to promote hypothermia under GA [17]. However, the time of Acetaminophen administration is not mentioned exactly, therefore it would be prudent to assume that no hypothermia occurred especially during emergence from GA.

Limitations to this study include sample size calculation due to the lack of national statistical data. The duration of the study was limited due to the inadvertent adverse events in October 2019 and Covid-19 pandemic conditions in the country. Moreover, despite randomization and standardized anesthesia regimen and warming techniques, it is difficult to account for variations between patients like body interactions to drugs, surgeries type and duration, and other factors contributing to IPH. Furthermore, effects of acetaminophen and IPH in neonates and older children may differ and it was not accounted for because our sample size is small to have an additional study group.

\section{Conclusion}

In conclusion, it is clear from this study that there is a significant transient hypothermia induced by IV Acetaminophen administration at T30 and T45. However, the discrepancy between the mean $T_{c}$ of Groups $\mathrm{P}$ and $\mathrm{N}$ is no longer significant at T60. Therefore, therapeutic doses of IV Acetaminophen can cause hypothermia in children thirty minutes after administration and hence should not coincide with emergence from GA. It is thus preferable to administer Acetaminophen one hour prior to emergence to avoid hypothermia at that critical phase. Prior studies relevant to this topic are very limited, where we did not find studies 


\section{Anaesthesia \& Critical Care Medicine Journal}

discussing the antipyretic effects of IV Acetaminophen in both adults and pediatrics population undergoing general anesthesia; therefore, further research is needed to address Acetaminophen induced hypothermia during GA and to investigate a better timing for administration, especially in the neonates and pediatrics population. Despite being widely used, Acetaminophen remains hardly understood.

\section{References}

1. McNicol E, Tzortzopoulou A, Cepeda M, Francia M, Farhat T, et al. (2011) Single-dose intravenous paracetamol or propacetamol for prevention or treatment of postoperative pain: a systematic review and metaanalysis. Br J Anaesth 106(6): 764-775.

2. (2020) IV Acetaminophen (Ofirmev). Pharmacy Times.

3. Russell FM, Shann F, Curtis N (2003) Mulholland K. Evidence on the use of paracetamol in febrile children. Bull World Health Organ 81(5): 367-372.

4. Berling I, Anscombe M, Isbister GK (2012) Intravenous paracetamol toxicity in a malnourished child. Clin Toxicol (Phila) 50(1): 74-76.

5. Van Tittelboom T, Govaerts-Lepicard M (1989) Hypothermia: an unusual side effect of paracetamol. Vet Hum Toxicol 31(1): 57-59.

6. McSwain J (2015) Perioperative hypothermia: Causes, consequences and treatment. World Journal of Anesthesiology 4(3): 58-65.

7. Sessler D (2020) UpToDate: perioperative-temperaturemanagement.

8. Hart SR, Bordes B, Hart J, Corsino D, Harmon D (2011) Unintended perioperative hypothermia. Ochsner J 11(3): 259-270.

9. Pai S, King A (2021) Up To Date: Emergence-fromgeneral-anesthesia.

10. Ellis TA, Edberg JL, Kumar N, Applefield DJ (2017) Delayed Emergence From Anesthesia: A Simulation Case for Anesthesia Learners. MedEdPORTAL 13: 10628.

11. Matika R, Ibrahim M, Patwardhan A (2016) The importance of body temperature: An anesthesiologist's perspective. Temperature (Austin) 4(1): 9-12.

12. (2005)" World Health Organization. Handbook: IMCI integrated management of childhood illness.

13. Ahangar N, Esam Z, Bekhradnia A, Ebrahimzadeh MA (2016) Hypothermic activity of acetaminophen; involvement of GABAA receptor, theoretical and experimental studies. Iran J Basic Med Sci 19(5): 470-
475.

14. Yi J, Lei Y, Xu S, Si Y, Li S, et al. (2017) Intraoperative hypothermia and its clinical outcomes in patients undergoing general anesthesia: National study in China. PLoS One 12(6): e0177221.

15. Pierce CA, Voss B (2010) Efficacy and safety of ibuprofen and acetaminophen in children and adults: a metaanalysis and qualitative review. Ann Pharmacother 44(3): 489-506.

16. Insler SR, Sessler DI (2006) Perioperative thermoregulation and temperature monitoring. Anesthesiol Clin 24(4): 823-837.

17. Visnjevac 0, Kocz R, Visnjevac T, Annam SK, Toufexis $G$ (2014) Medication safety: does intravenous acetaminophen promote perioperative hypothermia for total hip arthroplasty?. J Arthroplasty 29(11): 22302232.

18. Riley C, Andrzejowski J (2018) Inadvertent perioperative hypothermia. BJA Educ 18(8): 227-233.

19. Kurz A, Sessler DI, Lenhardt (1996) Perioperative normothermia to reduce the incidence of surgicalwound infection and shorten hospitalization. Study of Wound Infection and Temperature Group. N Engl J Med 334(19): 1209-1215.

20. Schmied H, Kurz A, Sessler DI, Kozek S, Reiter A (1996) Mild hypothermia increases blood loss and transfusion requirements during total hip arthroplasty. Lancet 347(8997): 289-292.

21. Winkler M, Akça O, Birkenberg B, Hetz H, Scheck T, et al. (2000) Aggressive warming reduces blood loss during hip arthroplasty. Anesth Analg 91(4): 978-984.

22. Pacifici GM, Allegaert K (2014) Clinical pharmacology of paracetamol in neonates: a review. Curr Ther Res Clin Expm 77: 24-30.

23. Agier MS, Chollet N, Bediou E, Boyer Gervoise M, Jonville-Bera AP (2019) Transient major hypothermia associated with acetaminophen: A pediatric case report and literature review. Arch Pediatr 26(6): 358-360.

24. Foster J, Mauger AR, Govus A, Hewson D, Taylor L (2017) Acetaminophen (Paracetamol) Induces Hypothermia During Acute Cold Stress. Clin Drug Investig 37(11):10551065. 